\title{
MORSE THEORY BY PERTURBATION METHODS WITH APPLICATIONS TO HARMONIC MAPS ${ }^{1}$
}

BY

\author{
K. UHLENBECK
}

\begin{abstract}
There are many interesting variational problems for which the PalaisSmale condition cannot be verified. In cases where the Palais-Smale condition can be verified for an approximating integral, and the critical points converge, a Morse theory is valid. This theory applies to a class of variational problems consisting of the energy integral for harmonic maps with a lower order potential.
\end{abstract}

The abstract Morse theory of Palais and Smale [9] can be applied with success to many problems in the calculus of variations to get existence theorems for stationary or critical points of integrals from topological information. However, there is a natural limitation in these applications in that the conditions are stated in terms of a fixed function space (which will usually be a Sobolev space). The integral must both be differentiable and satisfy a norm convergence property, which Palais and Smale call condition $\mathrm{C}$, in the same space of functions. In this article we introduce a perturbation method which circumvents this difficulty in some variational problems. This technique may be applied in particular to harmonic mappings, and we include this somewhat technical discussion in the last section. The author is grateful to G. K. Francis and R. S. Palais for a number of helpful conversations.

1. Palais-Smale Morse theory. In this article, $B$ will denote a Banach manifold with at least a $C^{2}$ structure, modeled on a Banach space with at least $C^{2}$ partitions of unity. We assume that this manifold is equipped with a $C^{1}$ Finsler structure in which the manifold is complete. If a function $f$ is defined and differentiable on $B$, $\left|d f_{x}\right|=\max _{0 \neq v \in T_{x}(B)}\left|d f_{x}(v)\right||v|_{x}^{-1}$ is well defined.

We say that the function $f$ satisfies Palais-Smale condition $\mathrm{C}$, if for any set $S \subset B$ on which $|f(S)|$ is bounded and $|d f(S)|$ is not bounded away from zero, the closure $\bar{S}$ contains a critical point of $f\left(\right.$ a point $x$ where $\left.d f_{x}=0\right)$. We have the following theorem due to Palais and Schwartz [10], [12].

(1.1) THEOREM. Let $B$ be a complete $C^{2}$ Finsler manifold (without boundary) and $f$ : $B \rightarrow R a C^{2}$ function satisfying Palais-Smale condition $C$. Then $f$ satisfies a Lusternik-Schnirelman theory. In particular

(a) If $f$ is bounded below it takes on its minimum on every component of $B$ and there are at least as many critical points as cat $(B)$.

Received by the editors March 13, 1979 and, in revised form, September 26, 1979. 1980 Mathematics Subject Classification. Primary 49F15; Secondary 58E05.

${ }^{1}$ Research supported in part by the National Science Foundation. 
(b) If there are no critical values on $[a, b]$, then $f^{-1}(-\infty, a)$ is a retraction of $f^{-1}(-\infty, b)$.

There are several possible extensions to a full Morse theory. If at a critical point $x \in B$, the Hessian $d^{2} f_{x}: T_{x}(B) \otimes T_{x}(B) \rightarrow R$ is a nondegenerate bilinear form, then $x$ is said to be a nondegenerate critical point, and the index is the dimension of the negative definite subspace. This, however, requires that $T_{x}(B)$ be a Hilbert space if the index happens to be finite (which is the interesting case in applications). The following extension can be used in a Banach space. We say that a critical point $x$ is weakly nondegenerate if there exists a coordinate neighborhood $U \subset T_{x}(M)$ of $x$ and a linear map $L: T_{x}(B) \rightarrow T_{x}(B)$ such that $d f_{v}(L v)>0$ for $v \in U, v \neq 0$. The linear map $L$ is assumed to have a spectrum which is bounded away from the imaginary axis and the index of $x$ is defined to be the dimension of the contracting subspace of $e^{L t}$. Tromba has a more general definition in terms of vector fields [14]. The following theorem is due to Palais and Smale in the case of nondegenerate critical points, and the author and Tromba in the weaker cases [14], [15].

(1.2) TheOREM. Let $B$ be a complete $C^{3}$ Finsler manifold with $C^{2}$ partitions of unity. If $f$ is a $C^{3}$ function which satisfies Palais-Smale condition $\mathrm{C}$, then $f$ satisfies a Morse theory. In particular, if $a<b$ are not critical values of $f$, and if the critical points in $f^{-1}(a, b)$ are nondegenerate or weakly nondegenerate, then $f^{-1}(-\infty, b)$ can be retracted onto $f^{-1}(-\infty, a)$ with handles adjoined corresponding to the critical points of $f$ in $f^{-1}(a, b)$. The dimensions of the handles correspond to the indices of the critical points.

Let us discuss the general framework of the applications of these theorems to the calculus of variations. We will consider $M$ to be a smooth compact manifold (with or without boundary), and $N$ a smooth finite dimensional manifold without boundary. We assume $N \subset R^{k}$ is an imbedding and $N$ is complete in $R^{k}$. Then the function manifolds which are conveniently available for the theory are the Sobolev spaces $L_{1}^{P}(M, N)=\left\{s: M \rightarrow R^{k}\right.$ a measurable function, $s(x) \in N$ almost everywhere, and $\left.\int_{M}|d s|^{p}+|s|^{p} d \mu_{M}<\infty\right\}$.

If $N$ is a linear subspace, $L_{1}^{p}(M, N)$ is a Banach space for $p>1$. For any $N \subset R^{k}$, the sets $L_{1}^{p}(M, N)$ are smooth Banach manifolds and not dependent on the imbedding of $N$ in $R^{k}$ only in the situation $p>\operatorname{dim} M$. In this case a natural complete Finsler structure is induced by the inclusion $L_{1}^{p}(M, N) \subset L_{1}^{p}\left(M, R^{k}\right)$, which is a Banach space equipped with a norm. In general, if we take $p$ large enough, an integral whose integrand depends smoothly on $(x, s(x), d s(x))$ will be smooth [11]. The difficulty arises in proving Palais-Smale condition C.

Suppose we illustrate this difficulty by considering a simple energy integral on functions on $M$. For $s$ a function on $M$, assuming $M$ has a metric and a measure, we can define an essentially quadratic integral

$$
E(s)=\int_{M}|d s(x)+v(x)|_{x}^{2}+b(x)(s(x))^{2} d \mu_{M}
$$


Here $v$ is a smooth one-form and $b$ a smooth function on $M$. In local coordinates the integrand will appear as

$$
g(x)\left(\sum_{i, j} g^{i j}(x)\left(\partial / \partial x_{i} s(x)+v_{i}(x)\right)\left(\partial / \partial x_{j} s(x)+v_{j}(x)+b(x) s^{2}(x)\right)\right) .
$$

This integral is smooth on the linear spaces $L_{1}^{p}(M, R)$ for all $p>2$. However, the integral (and its differential) give estimates only in $L_{1}^{2}(M, R)$, so we are forced to choose the function space $L_{1}^{2}(M, R)$ as the space used in the problem if we wish to use condition C. It is true that the critical points (functions) also lie in $L_{1}^{p}(M, R)$; in fact by the Weyl lemma the critical points lie in $C^{\infty}(M, R)$ and in all the Sobolev spaces. So the choice of $L_{1}^{2}(M, R)$ is artificial from the point of view of the partial differential equations for the critical points. This problem is not at all serious in this case, since the critical points are functions which satisfy a linear differential equation and are well understood.

However, if we had taken a more complicated integral on a vector-valued function $s(x)=\left(s^{1}(x), s^{2}(x), \ldots, s^{k}(x)\right)$,

$$
E(s)=\int_{M} L(x, s(x), d s(x)) d \mu_{M}
$$

where in local coordinates in $M$,

$$
L(x, s(x), d s(x))=\sum_{i, j, k, l} g(x) G_{k l}^{i j}(x, s(x))\left(\partial / \partial x_{i} s^{k}(x)\right)\left(\partial / \partial x_{j} s^{l}(x)\right)
$$

then this is a serious difficulty. Direct estimates from the integral are available only in $L_{1}^{2}\left(M, R^{k}\right)$ as before, so we might try to use this as the function space. However, the integral is not continuous on this function space, primarily because $s \in$ $L_{1}^{2}\left(M, R^{k}\right)$ is not sufficient to imply that $s$ is continuous and bounded almost everywhere. In fact, it is not clear that $E$ can be defined on all of $L_{1}^{2}\left(M, R^{k}\right)$. We would have to go to the smaller space $L_{1}^{p}\left(M, R^{k}\right)$ for $p>\operatorname{dim} M$ to get continuity of the integral, in which case the estimates needed to prove Palais-Smale condition $\mathrm{C}$ are not available.

In the next section we give a perturbation theory which will help avoid this difficulty. Instead of looking directly for the critical points of $f$, we add a small perturbation $\varepsilon g$ and look for the critical points of $f+\varepsilon g$, which does satisfy condition $C$. If we are lucky, the critical points of $f+\varepsilon g$ will converge to the critical points of $f$ as $\varepsilon \rightarrow 0$. For example, suppose we are trying to find the critical points of the function $f(x)=x^{-2}-x^{-1}$ on $R^{+}$. This function does not satisfy condition C. However, $f(x)+\varepsilon g(x)=x^{-2}-x^{-1}+\varepsilon x$ does satisfy condition $\mathrm{C}$ on $R^{+}$, and its minima converge to $x=2$, the minimum of $f$.

This is not particularly useful for functions on finite dimensional manifolds. However, in problems in the calculus of variations, the critical points are usually very smooth functions rather than arbitrary points in some Banach manifold. The equations for the critical points are elliptic systems, and the form of these equations gives a priori estimates on norms of the solutions which may very well be exactly what is needed to imply convergence. 
2. The abstract perturbation theory. Let $B$ be a complete $C^{2}$ Finsler manifold modeled on a Banach space which has $C^{2}$ partitions of unity and assume $f$ and $g$ are $C^{1}$ functions on $B$, and that $g$ is positive. We first state a simple theorem on existence of minima.

(2.1) THEOREM. Assume that $f+\varepsilon g$ is bounded below for all $\varepsilon>0$ and in addition

(a) $f+\varepsilon g$ satisfies Palais-Smale condition $\mathrm{C}$ for all $\varepsilon>0$,

(b) the set of critical points $\{x \in B: d(f+\varepsilon g)(x)=0,0 \leqslant \varepsilon<\delta, f(x)+\varepsilon g(x) \leqslant$ $k\}$ has compact closure in $B$ for all $k$ and some $\delta>0$.

Then $f$ is bounded below and takes on its minimum on each component of $B$.

Proof. $f+\varepsilon g$ is bounded below and satisfies condition C, so by (1.1), $f+\varepsilon g$ takes on its minimum on each component of $B$. Let $x(\varepsilon)$ be a point where $f+\varepsilon g$ takes on its minimum in one component $B_{i} . f(x(\varepsilon)) \leqslant f(x(\varepsilon))+\varepsilon g(x(\varepsilon)) \leqslant f(x)+$ $\varepsilon g(x)$ for all $x \in B_{i}$. Let $y$ be a limit point of $x(\varepsilon)$ as $\varepsilon \rightarrow 0$. This exists by condition (b) of the theorem.

$$
f(y)=\lim _{\varepsilon_{i} \rightarrow 0} f\left(x\left(\varepsilon_{i}\right)\right) \leqslant \lim _{\varepsilon_{i} \rightarrow 0}\left(f(x)+\varepsilon_{i} g(x)\right)=f(x)
$$

for all $x \in B_{i}$. Therefore $f(y)$ is the minimum of $f$ on $B_{i}$.

We now proceed to the more sophisticated version.

(2.2) TheOREM. Let $B$ be a complete $C^{3}$ Finsler manifold modeled on a separable Banach space with $C^{2}$ partitions of unity. Assume that $f, g \in C^{3}(B, R), f$ bounded below, $g$ positive and $|d g|$ bounded on sets on which $g$ is bounded. Suppose in addition

(a) $f+\varepsilon g$ satisfies the Palais-Smale condition $\mathrm{C}$ for $\varepsilon>0$,

(b) the critical set $\{x \in B: d(f+\varepsilon g)(x)=0, a \leqslant f(x)+\varepsilon g(x) \leqslant b, 0<\varepsilon<\delta\}$ has compact closure in $B$ for some $\delta>0$,

(c) $a<b$ are not critical values of $f$, and the critical points of $f$ with values in $(a, b)$ are nondegenerate.

Then $f^{-1}(-\infty, a)$ with handles adjoined corresponding to the critical points of $f$ with values in $(a, b)$ is a deformation retract of $f^{-1}(-\infty, b)$. The dimensions of the handles correspond to the dimensions of the indices of the critical points.

The theorem as stated essentially restricts the case to that of a Hilbert manifold unless there are no critical values in $(a, b)$. If a critical point of $f$ is nondegenerate, then there exists near the point a unique one-parameter family of critical points of $f+\varepsilon g$ smoothly parametrized by $\varepsilon$. With the regularity theorems available for critical points of integrals, this may follow from an implicit function theorem in some other Banach space. Therefore we state the theorem in a more applicable form.

(2.3) THEOREM. Theorem (2.2) is true if we replace (c) by (d).

(d) $a<b$ are not critical values of $f$, and if every critical point $x$ of $f$ with $a<f(x)<b$ is contained in a neighborhood $U$ such that the critical set of $f+\varepsilon g$ in $U$ for $0 \leqslant \varepsilon \leqslant \delta$ consists of a curve $x(\varepsilon), x(0)=x$, and these critical points are weakly nondegenerate of fixed index. 
Because (c) implies (d), it is sufficient to prove only (2.3). The fact that (c) implies (d) follows directly from the implicit function theorem in a Banach space, since the nondegeneracy of the Hessian is equivalent to a dual map being onto.

The proof of (2.3) is based on the Morse theory for Banach manifolds (1.2) and the fact that a function $h$ associated to $f+\varepsilon g$ with the same critical points does satisfy condition C. Let $B_{\varepsilon}^{b}=\{x \in B: f(x)+\varepsilon g(x)<b\}$.

(2.4) LEMMA. Assume that $f$ and $g$ satisfy the hypotheses of (2.2) except for condition (c). Then the implicit function $h(x)$ given by $b=f(x)+g(x) / h(x)$ satisfies Palais-Smale condition $\mathrm{C}$ on $\boldsymbol{B}_{0}^{b}$.

Proof. $h(x)=g(x) /(b-f(x))$ is defined, positive and differentiable for $b-$ $f(x)>0$. We have

$$
d h(x)=\left(d f(x)+h^{-1}(x) d g(x)\right) g(x)(b-f(x))^{-2} .
$$

So the critical points of $h$ are the critical points of $f+\varepsilon g$ with value $b$ in the range $\varepsilon>0$. By direct computation

$$
|d(f+\varepsilon g)(x)| \leqslant(b-f(x))^{2} g(x)^{-1}|d h(x)|+\left|\varepsilon-h(x)^{-1}\right||d g(x)| .
$$

Now suppose $h$ is bounded on a set $S$ and $|d h|$ is not bounded away from zero. From the hypotheses, it follows that $g(x)$ and therefore $|d g|$ are also bounded on $S$. Using the compactness of $h(x)^{-1}$ in $R$, we can extract a sequence $x(i) \in S$ such that $|d(f+\varepsilon g)(x(i))| \rightarrow 0$ for some $\varepsilon>0$. Since $f+\varepsilon g$ satisfies condition $\mathbf{C}$, the limit points of $x(i)$ contain a critical point of $f+\varepsilon g$ with critical value $b$, which is also a critical point of $h$.

(2.5) COROllary. If $f$ and $g$ satisfy the conditions of Theorem (2.2) except for (c) and there are no critical values of $f+\varepsilon g$ with value $b$ for $0<\varepsilon<\delta$, then $B_{\delta}^{b}$ is $a$ retract of $B_{\alpha}^{b}$ for $0 \leqslant \alpha<\delta$.

Proof. We check that $B_{e}^{b}=\left\{x \in B_{0}^{b}: h(x)<\varepsilon^{-1}\right\}$. The result follows from the equivalence of the critical points of $f+\varepsilon g$ with value $b$ and the critical points of $h$ and the application of (1.1) to the function $h$.

We are now able to construct a proof of (2.3) from (2.5). We assume $a<b<\infty$ are not critical values of $f$. Then there exists an interval $0<\varepsilon<\delta$ such that $a$ and $b$ are not critical values of $f+\varepsilon g$ in this interval, and the only critical points of $f+\varepsilon g$ lie in the one-parameter families of weakly nondegenerate critical points of $f+\varepsilon g$ near critical points of $f$. For suppose this is not the case. Then there exists a sequence $\varepsilon(i) \rightarrow 0$ and critical points of $f+\varepsilon(i) g$ with values on $[a, b]$ which do not lie in one-parameter families near the critical points of $f$. By condition (b) of (2.2), a subsequence $x(i) \rightarrow x$ for $x$ some critical point of $f$ with $a<x<b$. Since the critical points of $f+\varepsilon g$ are unique near $x$ by (d), it must be true that some of the points $x(i)$ lie in the one-parameter family for $x$. This contradicts the assumption.

It follows from (2.5) that $B_{\delta}^{b}$ is a retraction of $B_{0}^{b}$ and that $B_{\delta}^{a}$ is a retraction of $B_{0}^{a}$. However, from the Morse Theorem (1.2), $B_{\delta}^{a}$ with handles adjoined corresponding to the critical points of $f+\varepsilon g$ with values on $(a, b)$ is a retraction of $B_{\delta}^{b}$. 
By (d) these critical points correspond in a one-to-one fashion with the critical points of $f$ on $(a, b)$. This proves (2.3) for $b<\infty$.

In the case $b=\infty$, we choose an increasing sequence $b(i) \rightarrow \infty$ of values which are not critical values for $f$, and a decreasing sequence $\varepsilon(i) \rightarrow 0$ with the properties: (1) for $0 \leqslant \varepsilon \leqslant \varepsilon(i), b(i-1)$ and $b(i)$ are not critical values of $f+\varepsilon g$, and (2) the critical points of $f+\varepsilon g$ with values in $[a, b(i)]$ lie in the unique one-parameter families of weakly nondegenerate critical points of $f+\varepsilon g$ near the critical points of $f$. Then $B_{e(i)}^{b(i)}$ can be retracted onto $B_{e(i)}^{b(i-1)}$ with handles corresponding to the critical points of $f+\varepsilon(i) g$ with values on $(b(i-1), b(i))$. In addition, $B_{e(i-1)}^{b(i-1)}$ is a retraction of $B_{\varepsilon(i)}^{b(i-1)}$. This sequence of retractions gives the desired result for $B_{0}^{\infty}$.

3. An application to harmonic maps. If $M$ and $N$ are smooth compact finite dimensional Riemannian manifolds without boundary and $N \subset R^{k}$ an isometric imbedding, then an integral of the form

$$
F(s)=\int_{M}|d s|_{x}^{2}+a(s(x), x) d \mu_{M}
$$

can be defined for all differentiable maps $s: M \rightarrow N \subset R^{k}$. Here $a(y, x)$ is a smooth function on $N \times M,|d s|_{x}^{2}=\sum_{i=1}^{k}\left|d s^{i}\right|_{x}^{2}$ where $s=\left(s^{1}, s^{2}, \ldots, s^{k}\right)$ in the coordinates of $R^{k}$ and ||$_{x}$ denotes the Riemannian norm in $T_{x}^{*}(M)$. If $a=0$, this is the energy integral considered by Eells and Sampson [2] and the results are not new. For $a=0$, the critical maps of this integral are called harmonic maps, but in our application, all these maps are minima and the case $a=0$ does not illustrate the complete theorem.

We cannot apply Morse theory directly to this integral because of the difficulties explained in $\S 1$. It is also known that the minima are not necessarily taken on if $N$ has positive curvature, and a condition on the curvature of $N$ is necessary for a Morse theory.

It is clear that the integral $F$ is smooth on $C^{1}(M, N)$. At a critical point $s$, the Hessian is a bilinear form $H_{s}: T_{s}\left(C^{1}(M, N)\right) \otimes T_{s}\left(C^{1}(M, N)\right) \rightarrow R$ which cannot, of course, be nondegenerate. But there is a natural identification

$$
T_{s}\left(C^{1}(M, N)\right)=C^{1}\left(M, s^{*} T(N)\right)=\left\{u \in C^{1}\left(M, R^{k}\right): u(x) \in T_{s(x)}(N)\right\} .
$$

This Hessian will always extend to the larger Hilbert space $L_{1}^{2}\left(M, s^{*} T(N)\right)=\{u \in$ $L_{1}^{2}\left(M, R^{k}\right) ; u(x) \in T_{s(x)}(N)$ almost everywhere $\}$.

(3.2) We say that the critical point $s$ is nondegenerate if this extension to the Hilbert space is a nondegenerate bilinear form. The index of the critical point is the index of this bilinear form.

(3.3) THEOREM. Let $F$ be given as in (3.1) and assume in addition the sectional curvature of $N$ is nonpositive. If $a<b$ are not critical values of $F$ on $C^{1}(M, N)$ and all smooth critical maps $s: M \rightarrow N$ with $a<F(s)<b$ are nondegenerate, then $F^{-1}(-\infty, a) \subset C^{1}(M, N)$ with handles adjoined corresponding to the critical maps of $F$, the dimensions of the handles corresponding to the indices of the critical maps, is $a$ deformation retract of $F^{-1}(-\infty, b)$. 
The proof of the theorem is obtained by letting $f=F$ extended to the Banach manifold $L_{1}^{2 n}(M, N)$ and getting a Morse theory on this Banach space. Since the critical maps in $L_{1}^{2 n}$ are smooth, and $C^{1}$ is dense in $L_{1}^{2 n}$, the Morse theory will apply also to $C^{1}$ as well [7].

To get a Morse theory for $F$ on $L_{1}^{2 n}(M, N)$ we apply Theorem (2.3). The perturbing function we shall use is the functional $g(s)=G(s)=\int_{M}|d s|_{x}^{2 n} d \mu_{M}$, which is a very good functional for $L_{1}^{2 n}(M, N)$. The rest of the paper will contain a proof of the following two theorems:

(3.4) THEOREM. The hypotheses of (2.3) apply to $f=F$ and $g=G$ on the Banach manifold $L_{1}^{2 n}(M, N)$.

(3.5) THEOREM. Let $s$ be a nondegenerate critical map of $F$. Then condition (2.3)(d) is verified for $x=s$ in $L_{1}^{2 n}(M, N)$, that is there exist a neighborhood $U$ of $s$ in $L_{1}^{2 n}(M, N)$ and an interval $0 \leqslant \varepsilon \leqslant \delta$ such that the critical points of $F+\varepsilon G$ in $U$ consist of a curve $s(\varepsilon)$ of weakly nondegenerate critical points of index equal to the index of $s$.

4. Details of the application to harmonic maps. In this section we give a proof of Theorems (3.4) and (3.5). We have relied wherever possible on Palais' exposition of the calculus of variations [11], which contains many details of the framework. We also use the Sobolev spaces $L_{r}^{p}\left(M, R^{k}\right)$, the Sobolev manifolds $L_{r}^{p}(M, N)$ for $p r>\operatorname{dim} M$ and their tangent bundles $L_{r}^{p}\left(M, s^{*} T(N)\right)$, the Sobolev imbedding theorems, the basic $L^{p}$ interpolation theorems, as well as many continuity and multiplication results [11].

We deal with two compact Riemannian manifolds $M$ and $N \subset R^{k} .\langle$,$\rangle denotes$ the various inner products for $M,(\cdot)$ the various inner products for $N$, and $\langle;\rangle$ the combinations. The covariant derivative on $M$ will be denoted by $\nabla v=\left\{v_{\alpha ; \beta}\right\}$. We have chosen to rely heavily on the isometric imbedding of $N$ in $R^{k}$, although it is possible to carry out the proofs intrinsically. This imbedding generates a smooth map $P: N \rightarrow L\left(R^{k}, R^{k}\right)$, where $P(y)=\left\{P^{i j}(y)\right\}$ is the orthogonal projection in $R^{k}$ onto the tangent space $T_{y}(N)$. Covariant differentiation in $N$ is given by $P \circ d v=$ $\left\{P^{i j} v_{, h}^{j}\right\}$ in coordinates on $R^{k}$. We shall consistently use Greek indices for $M$, Latin indices for $N$. Because of the large number of contractions in the formulas, it will be necessary to use the index notation and summation convention.

We have collected a number of useful formulas. Summation signs are omitted, and because the indices for $N$ actually indicate coordinates in $R^{k}$, the identity is the metric tensor for $N$. We let $g^{\alpha \beta}$ denote the metric tensor for $M, R_{M}$ and $R_{N}$ the Riemannian curvature tensors for $M$ and $N$ respectively, and $S$ is the Ricci curvature on $M . Q=I-P$ is the orthogonal projection on the normal bundle at each point of $N$.

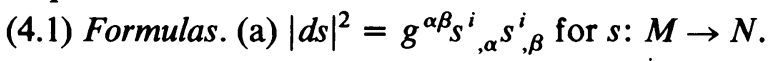

(b) $\nabla d f=\left\{f_{, \alpha ; \beta}\right\}$ for $f: M \rightarrow R, \nabla d s=\left\{s_{, \alpha ; \beta}^{i}\right\}$ for $s: M \rightarrow N$.

(c) $\operatorname{div} v=g^{\alpha \beta} v_{\alpha ; \beta}$, in particular div $d s=\left\{g^{\alpha \beta} s_{, \alpha ; \beta}^{i}\right\}$.

(d) $|w|^{2}=w^{2}$ indicates the norm in the appropriate metric. 
(e) $A(u, v)=Q(u \cdot d) v=Q(v \cdot d) u=\left\{Q^{i j} v^{k} u^{j}{ }_{, k}\right\}$ is an element of the normal bundle of $N$ when $u$ and $v$ are tangent vectors. $A$ is the second fundamental form of $N$.

(f) The Riemannian sectional curvature of $N$ is given by $\left(R_{N}(u, v) w \cdot z\right)=$ $(A(u, w) \cdot A(v, z))-(A(u, z) \cdot A(v, w))$.

With the above interpretations, the formulas which appear in the discussion of the critical points of $F+\varepsilon G$ will be unambiguous. We now return to the calculus of variations.

(4.2) Proposition. $F+\varepsilon G$ is smooth on $L_{1}^{2 n}(M, N)$ for $\varepsilon>0$. $F+\varepsilon G$ satisfies Palais-Smale condition $\mathrm{C}$ on $L_{1}^{2 n}(M, N)$ in a fixed, complete Finsler metric for all $\varepsilon>0$.

Proposition (4.2) is proved exactly in the fashion that Palais proves condition (C) for the functional $G$ in [11, Theorem 19.32].

(4.3) Proposition. The critical points of $F+\varepsilon G$ for $\varepsilon>0$ which are in $L_{1}^{2 n}(M, N)$ are smooth.

For $\varepsilon=0$, this result follows from the general regularity theory for solutions of elliptic systems. See in particular Morrey [5, §§1.11 and 6.2]. For $\varepsilon>0$ and $M$ and $N$ flat Riemannian manifolds, a proof of this regularity theorem can be found in [16]. A proof of this theorem for small $\varepsilon$, arbitrary $M$ and $N$ with nonpositive sectional curvature can be found in the appendix of this paper.

(4.4) Proposition. A critical map $s: M \rightarrow N$ of the function $F+\varepsilon G$ satisfies the partial differential equation

$$
\begin{aligned}
2 \operatorname{div}\left(\left(1+n \varepsilon|d s|^{2(n-1)}\right) d s\right)-(P d a) \circ s \\
\quad-2\left(1+n \varepsilon|d s|^{2(n-1)}\right) A \circ s\left(s_{, \alpha} ; s_{, \beta}\right) g^{\alpha, \beta}=0 .
\end{aligned}
$$

Proof. The differential of $F+\varepsilon G$ on $L_{1}^{2 n}\left(M, R^{k}\right)$ is easy to compute. For $\varphi \in L_{1}^{2 n}\left(M, R^{k}\right)$ we have

$$
d(F+\varepsilon G)(s) \varphi=\int_{M}\left(2\langle d \varphi ; d s\rangle\left(1+n \varepsilon|d s|^{2(n-1)}\right)+(\varphi \cdot d a(s))\right) d \mu .
$$

If $\varphi \in T_{s}\left(L_{1}^{2 n}(M, N)\right)$, then $P \circ s \varphi=\varphi$, and for any $\varphi, P \circ s \varphi \in T_{s}\left(L_{1}^{2 n}(M, N)\right)$. We conclude that $s$ is a critical point of $F+\varepsilon G$ on $L_{1}^{2 n}(M, N)$, if and only if $d(F+\varepsilon G)(s) P \circ s \varphi=0$ for all $\varphi \in L_{1}^{2 n}\left(M, R^{k}\right)$. Apply this to the equation for $d(F+\varepsilon G)$, integrate by parts in the first term, and use the fact that pointwise evaluation of $P$ gives an orthogonal projection. We end up with the Euler-Lagrange equation

$$
P \circ s\left(2 \operatorname{div}\left(1+n \varepsilon|d s|^{2(n-1)} d s\right)-d a(s)\right)=0 .
$$

But

$$
(P \circ s) \operatorname{div} v=\operatorname{div} v-(Q \circ s) \operatorname{div} v=\operatorname{div} v+(d Q \circ s ; v)
$$

where $v_{\alpha}^{i}=\left(1+n \varepsilon|d s|^{2(n-1)}\right) s_{, \alpha}^{i} . Q v_{\alpha}=0$, since $v_{\alpha}$ lies in the tangent space to $N$. 


$$
(d Q \circ s ; v)=Q_{, k}^{i j} s_{, \alpha}^{k} v_{\beta}^{j} g^{\alpha \beta}=-A\left(s_{, \alpha}, v_{\beta}\right) g^{\alpha \beta} .
$$

This shows that the two Euler-Lagrange equations are the same.

(4.5) LEMMA. If $f, \varphi$ and $\Psi$ are real-valued functions on $M$ which are sufficiently smooth, then we have

$$
\begin{aligned}
\operatorname{div}(\varphi d f) \operatorname{div}(\psi d f)= & (\varphi \psi)|\nabla d f|^{2}+\langle d \varphi, d f\rangle\langle d \psi, d f\rangle \\
& +\frac{1}{2}\left\langle d(\varphi \psi), d|d f|^{2}\right\rangle+\varphi \psi S(d f, d f)+\operatorname{div} W
\end{aligned}
$$

for some vector field $W$ on $M$.

Proof. Let $\sim$ signify equal modulo a pure divergence term. We write the left-hand side in index notation:

$$
\begin{aligned}
g^{\alpha \beta}\left(\varphi f_{, \alpha}\right)_{; \beta} g^{\gamma \delta}\left(\psi f_{, \gamma}\right)_{; \delta} & \sim-g^{\alpha \beta} g^{\gamma \delta}\left(\varphi f_{, \alpha}\right)\left(\psi f_{, \gamma}\right)_{; \delta ; \beta} \\
& =-g^{\alpha \beta} g^{\gamma \delta}\left(\left(\varphi f_{, \alpha}\right)\left(\psi f_{, \gamma}\right)_{; \beta ; \delta}+\left(R_{M}\left(x^{\beta}, x^{\delta}\right) d f\right)_{\gamma} f_{, \alpha} \varphi \psi\right) \\
& \sim g^{\alpha \beta} g^{\gamma \delta}\left(\varphi f_{, \alpha}\right)_{; \delta}\left(\psi f_{, \gamma}\right)_{; \beta}+\varphi \psi S(d f, d f) .
\end{aligned}
$$

Finally, the covariant differentiations in the last line are done out term-by-term, which yields the desired equation in a straightforward manner.

(4.6) Lemma. Let $s: M \rightarrow N$ be a smooth map. Then

$$
\begin{aligned}
&\left(\operatorname{div}(\varphi d s) \cdot\left(\operatorname{div}(\psi d s)-\psi A\left(s_{, \alpha}, s_{, \beta}\right) g^{\alpha \beta}\right)\right) \\
&= \varphi \Psi\left[|P \circ s \nabla d s|^{2}+S\left(d s^{i}, d s^{i}\right)-g^{\alpha \beta} g^{\gamma \delta}\left(R_{N}\left(s_{, \alpha}, s_{, \gamma}\right) s_{, \beta} \cdot s_{, \gamma}\right)\right] \\
&+(\langle d \varphi, d s\rangle \cdot\langle d \Psi, d s\rangle)+\frac{1}{2}\left\langle d(\varphi \psi), d|d s|^{2}\right\rangle+\operatorname{div} W .
\end{aligned}
$$

Proof. $(\operatorname{div}(\varphi d s) \cdot \operatorname{div}(\psi d s))$ is computed using the previous lemma on each coordinate $s^{i}$ and suming over $i .|\nabla d s|^{2}=|P \circ s \nabla d s|^{2}+|Q \circ s \nabla d s|^{2}$ and $|Q \circ s \nabla d s|^{2}=\left(A\left(s_{, \gamma}, s_{, \alpha}\right) \circ A\left(s_{, \gamma}, s_{, \beta}\right)\right) g^{\alpha \beta} g^{\gamma \delta}$. This is half the sectional curvature term. The other half comes from multiplying $\Psi$ by

$$
\begin{aligned}
\left(\operatorname{div}(\varphi d s) \cdot A\left(s_{, \alpha}, s_{, \beta}\right)\right) g^{\alpha \beta} & =\left(Q \circ s \operatorname{div}(\varphi d s) \cdot A\left(s_{, \alpha}, s_{, \beta}\right)\right) g^{\alpha \beta} \\
& =\varphi\left(A\left(s_{, \gamma}, s_{, \delta}\right) \cdot A\left(s_{, \alpha}, s_{, \beta}\right)\right) g^{\gamma \delta} g^{\alpha \beta} .
\end{aligned}
$$

Formula (4.1)(f) gives the curvature in terms of the second fundamental form $A$.

(4.7) LemMa. Let $s: M \rightarrow N$ be a smooth critical map of $F+\varepsilon G$. For simplicity of notation let $|d s|^{2}=q$. If $N$ has nonpositive sectional curvature, then for all $p>n / 2$

$$
\int_{M}\left\langle d q^{p}, d q^{p}\right\rangle d \mu\left\langle p^{2} /(2 p-1) K \int_{M} q^{2 p} d \mu .\right.
$$

The constant $K$ is independent of $p$ and depends on the size of the Ricci curvature $S$ and the derivatives of the projection $P$ and the function $a$ on $N$.

Proof. Let $\psi=\left(1+\varepsilon n q^{n-1}\right)$. Then from (4.4) we get

$$
\operatorname{div}(\psi d s)-\psi A\left(s_{, \alpha}, s_{, \beta}\right) g^{\alpha \beta}=\frac{1}{2}(P d a) \circ s .
$$


Apply (4.6) and integrate:

$$
\begin{aligned}
\int_{M}\left(\varphi \psi \left\{-R_{N}\left(s_{, \alpha}, s_{, \gamma}\right)\left(s_{, \beta} \cdot s_{, \gamma}\right)\right.\right. & \left.g^{\alpha \beta} g^{\gamma \delta}+|P \circ s \nabla d s|^{2}+S\left(d s^{i}, d s^{i}\right)\right\} \\
& \left.+\frac{1}{2}(d(\varphi \psi), d q)+(\langle d \varphi, d s\rangle \cdot\langle d \Psi \cdot d s\rangle)\right) d \mu \\
= & \frac{1}{2} \int_{M}(\operatorname{div}(\varphi d s) \cdot(P d a) \circ s) d \mu \\
= & -\frac{1}{2} \int_{M} s_{, \beta}^{j}\left((P d a)_{j} \cdot s_{, \alpha}\right) g^{\alpha \beta} d \mu .
\end{aligned}
$$

Let $K_{1}=\max _{y \in N}\left|(P \circ d a)_{j}(y)\right|, K_{2}=\max _{x \in M}(-S(v, v) /(v, v))$ and $\Psi \varphi=q^{2 p-1}$. For this choice of $\varphi$ with $p \geqslant n / 2, d / d q \varphi \geqslant 0$.

If the sectional curvature is nonpositive, the equality can be replaced by the inequality

$$
\begin{aligned}
& \frac{2 p-1}{2 p^{2}} \int_{M}\left\langle d q^{p}, d q^{p}\right\rangle d \mu=\frac{1}{2} \int_{M}\langle d(\varphi \psi), d q\rangle d \mu \\
& \quad \leqslant K_{1} \int_{M} \varphi q d \mu+K_{2} \int_{M} \varphi \Psi d \mu \leqslant\left(K_{1}+K_{2}\right) \int_{M} q^{2 p} d \mu .
\end{aligned}
$$

Let $K=K_{1}+K_{2}$.

It is at this point evident where the condition on the sectional curvature will come in. From now on we assume that the sectional curvature of $N$ is nonpositive. The proof of the following lemma is similar to some proofs of Moser in [6] and the proof of the regularity theorem in [16].

(4.8) Proposition. Let $s$ be a smooth critical point of $F+\varepsilon G, \varepsilon>0$. Then there exists a constant $C$ which depends only on the geometry of $M$ and $N$ such that

$$
\max _{x \in M} q(x)<C \int_{M} q(x) d \mu .
$$

Proof. Fix any $p>n, \nu=n /(n-1)$ and $0<\alpha=(\nu-1) /(2 p \nu-1)<1$. From the continuous Sobolev imbedding $L_{1}^{2}(M) \subset L^{2 v}(M)$ there exists a constant $c$ independent of $p$ such that

$$
\left(\int_{M} q^{2 p \nu} d \mu\right)^{1 / \nu}<c \int_{M}\left(\left\langle d q^{p}, d q^{p}\right\rangle+q^{2 p}\right) d \mu
$$

Using interpolation on the $L^{p}$ spaces, we also know that $\int_{M} q^{2 p} d \mu<$ $\left(\int_{M} q^{2 p v} d \mu\right)^{(1-\alpha) / v}\left(\int_{M} q d \mu\right)^{\alpha 2 p}$. By enlarging the constant in (4.7) slightly we find

$$
\int_{M}\left\langle d q^{p}, d q^{p}\right\rangle d \mu \leqslant K(p+1) \int_{M} q^{2 p} d \mu .
$$

If these three inequalities are combined and rearranged, we are able to derive an inequality of the form $\left(\int_{M} q^{2 p} d \mu\right)^{1 / 2 p}<c(p) \int_{M} q d \mu$ which is valid for all $p>n$. However, we are not quite finsihed because $\lim _{p \rightarrow \infty} c(p)=\infty$. 
Let $p(i)=2 \nu^{i} p$ for some $p>n$. From two of the three initial inequalities given above we arrive at one of the form

$$
\left(\int_{M} q^{p(i+1)} d \mu\right)^{1 / \nu} \leqslant c(K p(i)+1)\left(\int_{M} q^{p(i)} d \mu\right) .
$$

We check by elementary methods that the infinite product $\Pi_{i-0}(c(K p(i)+1))^{1 / p(i)}$ $=k$ is convergent. This allows us to conclude by induction on $i$ that

$$
\begin{aligned}
\max _{x \in M} q(x) & =\lim _{i \rightarrow \infty}\left(\int_{M} q^{p(i)} d \mu\right)^{1 / p(i)} \\
& \leqslant k\left(\int_{M} q^{2 p} d \mu\right)^{1 / 2 p} \leqslant k c(p) \int_{M} q d \mu .
\end{aligned}
$$

This completes the proof of (4.8).

(4.9) THEOREM. Let $S_{\delta}^{b}$ be the set of critical points of $F+\varepsilon G$ for $0<\varepsilon \leqslant \delta$ and $F(s) \leqslant F(s)+\varepsilon G(s) \leqslant b$. Then $S_{\delta}^{b}$ is compact in the Sobolev manifold $L_{1}^{2 n}(M, N)$.

Proof. In (4.8) we showed that $\max _{x \in M} q(x)$ is uniformly bounded for all $s \in S_{\delta}^{b}$, as it is easy to see that $\int_{M} q d \mu$ is uniformly bounded over the same set. Due to the continuity of the integral, $S_{\delta}^{b}$ is certainly closed in $L_{1}^{2 n}(M, N)$. It is sufficient to show sequential compactness, and we can assume for any sequence $s(i)$ that $s(i)$ is a critical point of $F+\varepsilon(i) G$ where the sequence of real numbers $\varepsilon(i)$ converges.

There are two cases. The easy one to deal with is when $\varepsilon(i) \rightarrow \varepsilon>0$. Then $|(d F+\varepsilon d G) s(i)|=|(\varepsilon-\varepsilon(i) d G s(i))| \rightarrow 0$ and we may use the valid condition $\mathrm{C}$ for $F+\varepsilon G$ with $\varepsilon>0$. More difficulty naturally occurs when $\varepsilon(i) \rightarrow 0$. Then we may assume $\varepsilon(i)=\varepsilon$ is as small as we need it to be. We simply rewrite the EulerLagrange equations (4.4) for $s$ treating $s: M \rightarrow N \subset R^{k}$ as a vector-valued function on $M$, and putting the lower order terms and the small term with $\varepsilon$ in front on the right-hand side of the equation. This yields an equation of the form

$$
\Delta s=\varepsilon T \cdot \nabla d s+B .
$$

$T$ and $B$ are tensors which depend on $(x, s(x), d s(x))$ and due to the boundedness of these jets these tensors $T$ and $B$ are uniformly bounded for all $s \in S_{\delta}^{b}$.

Denote an $L_{r}^{p}\left(M, R^{k}\right)$ norm by \|\|$_{(r, p)}$. Using the basic properties of the elliptic Laplace operator on $M[1]$, we find

$$
\begin{aligned}
\|s\|_{(2,2 p)} & \leqslant c(p)\left(\|\Delta s\|_{(0,2 p)}+\|q\|_{(0, p)}+\|s\|_{(0,2 p)}\right) \\
& \leqslant c(p)\left(\varepsilon\|T\|_{(0, \infty)}\|s\|_{(2,2 p)}+\|B\|_{(0, \infty)}+\|q\|_{(0, p)}+\|s\|_{(0,2 p)}\right),
\end{aligned}
$$

where $c(p)$ is a constant depending on properties of the Laplace operator on $M$ and the choice of $p$. We may assume that $\varepsilon$ is arbitrarily small, in particular that $\varepsilon c(p)\|T\|_{(0, \infty)} \leqslant \frac{1}{2}$.

We have already shown that the other norms involving $s$ are bounded for $s \in S_{\delta}^{b}$. We are finally able to arrive at a uniform bound for $\|s\|_{(2,2 p)}$. The only restriction on $p$ is $1<2 p<\infty$, so from the compact imbedding theorems, the set $S_{\delta}^{b}$ is precompact in $C^{1}(M, N)$ as well as in $L_{1}^{2 n}(M, N)$ for small enough $\delta$. 
This completes the proof of (4.9), and the demonstration of (3.4) is complete. We still have to prove (3.5) and deal with nondegeneracy. A slight modification of the proof just given in (4.9) will help here. We have just shown that for a sequence $\{s(i)\}, s(i) \in S_{\varepsilon(i)}^{b}$ with $\varepsilon(i) \rightarrow 0, s(i) \in L_{2}^{2 p}(M, N) \subset L_{2}^{2 p}\left(M, R^{k}\right)$ is bounded for $1<2 p<\infty$. If $\{s(i)\}$ is convergent in $L_{1}^{2 n}(M, N)$, then it will be convergent in $C^{1}(M, N)$. Since $\Delta s(i)=\varepsilon(i) T_{i} \nabla d s+B_{i}$, where $T_{i}$ is uniformly bounded, $\varepsilon(i) \rightarrow 0$, and $B_{i}$ depends smoothly on the 1-jet of $s$, then $B_{i}$ converges in $C^{0}\left(M, R^{k}\right)$ it follows that $\{\Delta s(i)\}$ is Cauchy in $L^{2 n}\left(M, R^{k}\right)$. It follows that $\{s(i)\}$ converges in the manifold $L_{2}^{2 n}(M, N)$. We restate this corollary of the proof in terms of neighborhoods.

(4.10) Corollary. Let $s$ be a critical point of $F$ and $U_{0}$ a neighborhood of $s$ in $L_{2}^{2 n}(M, N)$. Then there exists a neighborhood $U$ of $s$ in $L_{1}^{2 n}(M, N)$ and a $\delta>0$ such that $S_{\delta}^{\infty} \cap U \subset U_{0}$.

Recall the definition of nondegenerate which we are using for the critical points of these functionals. The tangent bundle to an element $s \in L_{r}^{p}(M, N) \subset$ $L_{r}^{p}\left(M, R^{k}\right)$ consists of sections $\left\{u \in L_{r}^{p}\left(M, R^{k}\right): u(x) \in T_{s(x)}(N)\right\}=$ $L_{r}^{P}\left(M, s^{*} T(N)\right)$, according to the definition of the pull-back bundle $s^{*} T(N)$. Since all critical maps $s \in S_{\delta}^{b}$ will be smooth, the pull-back bundle $\eta=s^{*} T(N)$ is a smooth bundle and the Banach space of sections $L_{r}^{p}(\eta)$ is defined for all $1<p<$ $\infty$ and all $-\infty<r<\infty$. Locally we may regard $F+\varepsilon G$ as a smooth functional on $L_{r}^{p}(\eta)$ for $r \geqslant 1$ and $p \geqslant 2 n$. Without looking at the precise form of the functional, the differential of $F+\varepsilon G$ is a smooth map from $L_{r}^{p}(\eta)$ to $L_{r}^{p}(\eta)^{*}$ which we may identify with the space of distributions $L_{-r}^{q}\left(\eta^{*}\right)$. However, if the exact coordinate description of $F+\varepsilon G$ is carefully written out, we can do much better, and treat the entire problem as a problem in elliptic operators.

(4.11) LEMMA. $d(F+\varepsilon G)$ is a nonlinear elliptic operator of order two from $\eta$ to $\eta^{*}$ for $\varepsilon \geqslant 0$ which extends to a smooth map $d F+\varepsilon G:[0, \infty) \times L_{2}^{2 n}(\eta) \rightarrow L_{0}^{2 n}\left(\eta^{*}\right)$. $H_{\varepsilon, s}=d^{2}(F+\varepsilon G)_{s}$ extends to a symmetric bilinear form on $L_{1}^{2}(\eta)$ which is continuous for $(\varepsilon, s) \in R^{+} \times L_{2}^{2 n}(\eta)$.

Proof. This is proved by writing out the integrand and its various derivatives in coordinates in $M$ and $N$. The integrand in the functional will be $\left(q+\varepsilon q^{n}+a(s)\right) g$, where $q=\sum_{i, j, \alpha, \beta} g^{\alpha \beta}(s) G_{i j}(s(x)) s_{, \alpha}^{i} s_{, \beta}^{j}$ and $g=\operatorname{det}\left\{g_{\alpha, \beta}\right\}$. The differentials are computed and shown to be smooth for this type of integral by Palais [11, §16].

(4.12) Proposition. If $s$ is a nondegenerate critical point of $F$, then there exists $a$ neighborhood $U$ of $s$ in $L_{1}^{2 n}(\eta)$ and an interval $[0, \delta)$ such that $d(F+\varepsilon G)^{-1}(0) \cap U$ $=s(\varepsilon)$ is a continuous curve for $0 \leqslant \varepsilon<\delta . H_{\varepsilon, s(e)}=d^{2}(F+\varepsilon G)_{s(\varepsilon)}$ is a continuous family of nondegenerate bilinear forms on $L_{1}^{2}(\eta)$ with constant index for $0 \leqslant \varepsilon<\delta$.

Proof. If $s$ is nondegenerate, $H_{0}$ is a nondegenerate bilinear form, then $d^{2} F_{s}$ is an invertible linear elliptic differential operator both from $L_{1}^{2}(\eta) \rightarrow L_{-1}^{2}\left(\eta^{*}\right)$ and from $L_{2}^{2 n}(\eta) \rightarrow L_{0}^{2 n}\left(\eta^{*}\right)$. We can then apply the implicit function to $d(F+\varepsilon G)$ : $L_{2}^{2 n}(\eta) \rightarrow L_{0}^{2 n}\left(\eta^{*}\right)$ to get a neighborhood $U_{0}$ of $s$ in $L_{2}^{2 n}(\eta)$ and a $\delta$ such that 
$S_{\delta} \cap U_{0}=\{s(\varepsilon), 0<\varepsilon<\delta\}$. For a possibly smaller $\delta$, there exists $U \subset L_{1}^{2 n}(\eta)$ such that $S_{\delta} \cap U=S_{\delta} \cap U_{0}=\{s(\varepsilon), 0<\varepsilon<\delta\}$. By the composition rule, $H_{\varepsilon, s(e)}$ is a continuous curve of bilinear forms on $L_{1}^{2}(\eta)$. Since the nondegenerate bilinear forms are open sets of fixed index on $L_{1}^{2}(\eta), H_{e, s(e)}$ will be nondegenerate of the index of $H_{0}$ for some interval about zero. Again, we may have to take a smaller value of $\delta$ to complete the proof.

The idea of weak-nondegeneracy was developed to treat exactly the type of integral of $F+\varepsilon G$. The following theorem is a direct application of 5.5 in [15].

(4.13) THEOREM. If $u \in L_{2}^{2 n}(\eta) \subset C_{1}^{\alpha}(\eta)$ is a critical point of $F+\varepsilon G$, and the Hessian $d^{2} F+\varepsilon G_{u}=H_{\varepsilon, u}$ extends to a nondegenerate bilinear form on $L_{1}^{2}(\eta)$, then $u$ is a weakly nondegenerate critical point.

The proof of (3.5) is now an immediate consequence of (4.12) and (4.13). There is a neighborhood $U$ of $s$ such that the set of critical points of $(F+\varepsilon G)$ for $0<\varepsilon<\delta$ in $U$ is a single critical point $s(\varepsilon)$. This is a weakly nondegenerate critical point of the index of $s(0)=s$. This is precisely what we needed to finish (3.5).

Appendix. The regularity theorem. In (4.3) we state that the solutions to the Euler-Lagrange equations (*) are smooth and refer to a general theory of the regularity of solutions to this type of equation. In fact, a much simpler proof of the regularity is valid for small $\varepsilon$, and we present this proof in the following pages. Recall that $q=|d s|^{2}$ as in (4.1)(a) and let $\Psi=1+\varepsilon q^{n-1}$ as in the proof of (4.7). The Euler-Lagrange equations appear in the form (*) in (4.4) and (**) in (4.9).

(A.1) Proposition. Let $s \in L_{1}^{2 n}(M, N)$ be a solution of (*). Then $s \in L_{2}^{2}(M, N)$, $q^{(n-1) / 2} \nabla d s \in L^{2}\left(M, R^{k n^{2}}\right)$ and $q \in L^{n^{2} /(n-2)}(M)$.

Proof. We may apply Theorem 1.11.1, p. 36 of Morrey [5] to the Euler-Lagrange equations. The first two statements follow from this theorem, and the last from the Sobolev imbedding of $q^{n / 2} \in L_{1}^{2}(M) \subset L^{2 n /(n-2)}(M)$.

(A.2) Proposition. Let $s \in L_{1}^{2 n}(M, N)$ be a solution of (*). Then $q \in L^{r}(M)$ for all $r<\infty$.

Proof. We give a proof which is valid for the sectional curvature of $N$ nonpositive, although with care the sectional curvature term of $N$ could be included in the estimate when it is positive. We apply Lemma (4.6) with $\Psi=1+$ $\varepsilon n q^{n-1}$, but with $\varphi=\min \left(q^{2 p-1} / \Psi, e\right)$.

From (A.1) we have sufficient differentiability to perform the integrations. The terms which are integrals of $\varphi \Psi|P \nabla q|^{2},(\langle d \varphi, d\rangle s \cdot\langle d \Psi, d\rangle s)$ and the sectional curvature of $N$ are all positive, so we get the inequality

$$
\frac{1}{2} \int_{M}\langle d(\varphi \Psi), d q\rangle d \mu \leqslant K_{1} \int_{M} \varphi d \mu+K_{2} \int_{M}(\varphi \Psi) q d \mu .
$$

If $q \in L^{2 p}$, the left-hand side of this inequality is bounded by $\left(K_{1}+K_{2}\right) \int_{M} q^{2 p} d \mu$ independently of the choice of $e$ in the definition of $\varphi$. By letting $e \rightarrow \infty$, we get a 
bound for $\int_{M}\left\langle d\left(q^{2 p-1}\right), d q\right\rangle d \mu$, which from the Sobolev imbedding theorem implies that $q \in L^{2 p n /(n-1)}(M)$. By induction on $r$, we prove $q \in L^{r}(M)$ for all $r$ by this process.

(A.3) Corollary. Let $s \in L_{1}^{2 n}(M, N)$ be a solution to (*) and assume that $N$ has nonpositive sectional curvature. Then $s \in L_{1}^{\infty}\left(M, R^{k}\right)$, and there exists a constant $K$ such that $\|q\|_{\infty} \leqslant K \int_{M} q d \mu$.

Proof. The proof of (4.8) depends only on the existence of the integrals on the left-hand side of the equation, which follows from our construction in (A.2), so we may apply (4.8).

We now proceed to analyze the next step in the a priori estimates more carefully, and show how to get from equation $(*)$ to one of the form $(* *)$ in detail. We have demonstrated sufficient differentiability to differentiate out the first term in (*):

$$
d^{*} \Psi d s=\Psi\left(\Delta s+2 \varepsilon n(n-1) q^{(n-2)} / \Psi\langle\langle\nabla d s ; d s\rangle \cdot d s\rangle\right) .
$$

We define the second order operator with bounded measurable coefficients

$$
\begin{aligned}
L(u) & =2 n(n-1) q^{(n-2)} / \Psi\langle\langle\nabla d u ; d s\rangle, d s\rangle \\
& =2 n(n-1) q^{(n-2)} / \Psi\left(s_{, \alpha} s_{, \beta}^{k} g^{\alpha \delta} g^{\beta \gamma} u_{\delta ; \gamma}^{k}\right) .
\end{aligned}
$$

Then the coefficients of $L$ have been uniformly bounded by $2 n(n-1)\|q\|_{\infty}^{n}$, and the Euler-Lagrange equations (*) appear as (**) when divided by $\Psi$.

$$
\Delta s+\varepsilon L(s)+A(s)(d s, d s)+(P d a)(s) / \Psi=0 .
$$

(A.4) THEOREM. Let $E(s) \leqslant b$ and $s \in L_{1}^{2 n}(M, N)$ be a solution of (*), where $N$ has nonpositive sectional curvature. Then there exists an $\varepsilon(b)$ such that for $\varepsilon<\varepsilon(b)$, we have $s \in L_{2}^{2 n}\left(M, R^{k}\right)$. Moreover, the $L_{2}^{2 n}$ norm of $s$ is uniformly bounded in this case.

Proof. Since $E(s) \leqslant b$, we have $\int_{M} q d \mu \leqslant b+\mu(M)\|a\|_{\infty}=b^{\prime}$. From (A.3) we have the uniform estimate $\|q\|_{\infty} \leqslant K b^{\prime}$, and the coefficients of $L$ are uniformly bounded by $2 n(n-1)\left(K b^{\prime}\right)^{n}$. It follows that the norm of $L$ as a map from $L_{2}^{p}\left(M, R^{k}\right)$ to $L^{p}\left(M, R^{k}\right)$ is bounded by $2 n(n-1)\left(K b^{\prime}\right)^{n}=K(b)$. Let $c(p)^{-1}$ be the norm of $(\Delta-1)^{-1}$ as a map from $L^{p}(M)$ to $L_{2}^{p}(M)$. Choose $\varepsilon(b)$ so that, for $p=2$ and $p=2 n, \varepsilon(b) c(p) K(b)<\frac{1}{2}$.

Then the equation

$$
(\Delta-I) u+\varepsilon L u=-(s+A(s)(d s, d s)+P d a(s)) / \Psi
$$

has the unique solution $u=s$ in $L_{2}^{2}\left(M, R^{k}\right)$ from (*) and the fact that the operator $\Delta-I+\varepsilon L$ is invertible as a map from $L_{2}^{2}\left(M, R^{k}\right)$ to $L^{2}\left(M, R^{k}\right)$. We already know $s \in L_{2}^{2}\left(M, R^{k}\right)$. This same operator is invertible from $L_{2}^{2 n}\left(M, R^{k}\right)$ to $L^{p}\left(M, R^{k}\right)$, so there is a solution $u \in L_{2}^{2 n}\left(M, R^{k}\right)$. However, this solution must be $s$ from the uniqueness in $L_{2}^{2}\left(M, R^{k}\right)$. With this choice of $\varepsilon(b)$, we have for $\varepsilon \leqslant \varepsilon(b)$,

$$
\begin{aligned}
\|s\|_{(2,2 n)} & =\left\|(\Delta-I+\varepsilon L)^{-1}(s+A(s)(d s, d s)+P d a / \Psi)\right\|_{(0,2 n)} \\
& \leqslant 2 c(2 n)^{-1}\|s+A(s)(d s, d s)+P d a / \Psi\|_{(0,2 n)} .
\end{aligned}
$$


(A.5) Corollary. Let $E(s) \leqslant b$, and $s \in L_{1}^{2 n}(M, N)$ be a solution of (*), where $N$ has nonpositive sectional curvature. Then there exists $\varepsilon(b)>0$, such that for $0<\varepsilon<$ $\varepsilon(b), s \in C^{\infty}(M, N)$.

Proof. In (A.4) we prove the solutions are in $L_{2}^{2 n}\left(M, R^{k}\right)$, which from the Sobolev imbedding theorem implies that the solutions have Hölder continuous derivatives with exponent $\frac{1}{2}$. The differentiated equations (*) will form a second order linear elliptic system for $d s$ in divergence form, and the coefficients will be at least Hölder continuous, so we may apply the Schauder theory to get regularity.

\section{REFERENCES}

1. S. Agmon, The $L_{p}$ approach to the Dirichlet problem, Ann. Scuola Norm. Sup. Pisa 13 (1959), 405-448.

2. J. Eells, Jr. and J. H. Sampson, Harmonic mappings of Riemannian manifolds, Amer. J. Math. 86 (1964), 109-160.

3. R. Hamilton, Harmonic maps of manifolds with boundary, Lecture Notes in Math.., vol. 471, Springer-Verlag, Berlin and New York, 1975.

4. P. Hartman, On homotopic harmonic maps, Canad. J. Math. 19 (1967), 673-687.

5. C. B. Morrey, Multiple integrals in the calculus of variations, Springer-Verlag, Berlin and New York, 1966.

6. J. Moser, A new proof of de Giorgi's theorem for elliptic differential equations, Comm. Pure Appl. Math. 13 (1960), 457-468.

7. R. Palais, Homotopy theory of infinite dimensional manifolds, Topology 5 (1966), 1-16.

8. __ Morse theory on Hilbert manifolds, Topology 2 (1963), 299-340.

9. R. Palais and S. Smale, A generalized Morse theory, Bull. Amer. Math. Soc. 70 (1964), 165-171.

10. R. Palais, Lusternik-Schnirelman theory on Banach manifolds, Topology 5 (1966), 115-132.

11. F__ Foundations of global non-linear analysis, Benjamin, New York, 1968.

12. J. Schwartz, Generalizing the Lusternik-Schnirelman theory of critical points, Comm. Pure Appl. Math. 17 (1964), 307-315.

13. S. Smale, Morse theory and a non-linear generalization of the Dirichlet problem, Ann. of Math. 80 (1964), 382-396.

14. A. Tromba, A general approach to Morse theory, J. Differential Geom. 12 (1977), 47-86.

15. K. Uhlenbeck, Morse theory on Banach manifolds, J. Funct. Anal. 10 (1972), 430-445.

16. __ Regularity for a class of non-linear elliptic systems, Acta Math. 138 (1977), 219-240.

17. Harmonic maps; A direct method in the calculus of variations, Bull. Amer. Math. Soc. 76 (1970), $1082-1087$.

Department of Mathematics, University of Illinois at Chicago Circle, Chicago, Illinois 60680 